\title{
EL TEXTO BUSCADO \\ Los textos auxiliares en la enseñanza de traducción
}

\section{Christiane Nord*}

\begin{abstract}
RESUMEN: El presente articulo analiza los factores y los componentes del proceso de traducción desde una perspectiva pedagógica, particularmente, con respecto a las habilidades y a los campos de conocimiento que deben desarrollarse en función de la formación de traductores profesionales. Se busca mostrar que la enseñanza de la traducción, en varios aspectos, aún tiene sus bases en el intercambio de experiencias profesionales entre profesores y traductores que ya poseen una práctica, asi como también en los consejos prácticos dados en clase. Los diccionarios y las gramáticas tradicionales no traen informaciones apropiadas o incluso suficientes para permitir soluciones satisfactorias paravarios problemas de traducción, especialmente en lo que se refiere a convenciones estilísticas, textuales y las vinculadas al género o, también, en relación a los tecnoletos y a la terminología. A partir de esa premisa, la autora sugiere unaveriedad de textos auxiliares, entre los cuales se cuentan los llamados 'textos paralelos', que pueden ser útiles para el análisis del texto de la lengua original y para la producción del texto de la lengua terminal, tanto para traductores en fase de entrenamiento como para profesionales.
\end{abstract}

PALABRAS CLAVE: formación de traductore; conocimiento y habilidade; textos paralelos.

RESUMO: O presente artigo analisa os fatores e constituintes do processo de tradução de um ponto de vista pedagógico, particularmente no que se refere às habilidades e campos do

(*) Escuela Superior Politécnica (Fachhochschule) de Magdeburg/Alemania, Departamento de Comunicación especializada y Traducción. 
conhecimento a serem desenvolvidos na formação de tradutores profissionais. Procura-se demonstrar que o ensino de tradução, sob vários aspectos, ainda tem suas bases sobretudo na troca de experiências profissionais entre professores e tradutores que já dispõem de alguma prática, bem como na oferta de conselhos práticos em sala de aula. Os dicionários e as gramáticas tradicionais não veiculam informações apropriadas ou suficientes para permitirem soluções satisfatórias para vários problemas de tradução, especialmente no que respeita a convencões estilísticas, textuais e ligadas ao gênero, ou no que respeita aos tecnoletos e à terminologia. Partindo dessa premissa, a autora sugere uma variedade de textos auxiliares, dentre os quais os assim-chamados textos paralelos, que podem ser úteis na análise do texto-fonte e na produção do textometa, tanto para tradutores em fase de treinamento, quanto para profissionais.

PALAVRAS CHAVE: formação de tradutores; conhecimento $e$ habilidades; textos paralelos.

El siguiente esquema muestra el proceso de traducción visto desde una perspectiva didáctica (cf. la versión alemana en Nord 1996b: 314).

TradTerm, 4(1), $1^{\circ}$ semestre de 1997, p. 101-124 
1. El proceso de traducción (desde una perspectiva didáctic

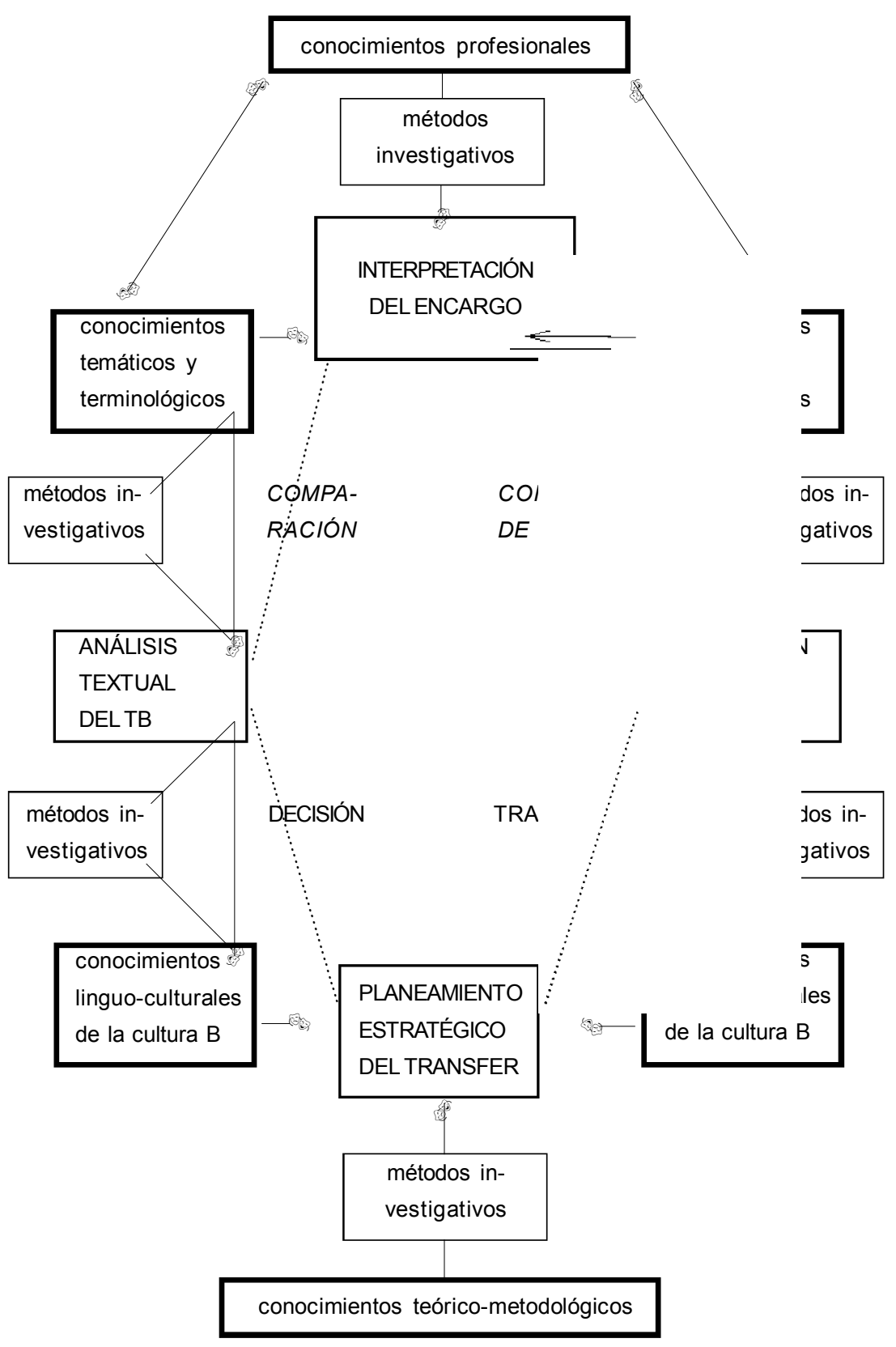

TradTerm, 4(1), $1^{\circ}$ semestre de 1997, p. 101-124 
El proceso se inicia con el encargo de traducción proporcionado por el cliente del traductor o inicador. Este encargo suele incluir un texto que llamaremos texto base o TB. Este término me parece más apropiado que las denominaciones usuales texto original (algunos textos base no son ni siquiera originales sino textos ya traducidos de otra lengua) o texto de partida (este término da la impresión de un proceso lineal que más o menos automáticamente, como los carriles de un tranvía, conduce de un punto de partida a un punto determinado de llegada) para caracterizar su función de servir de base material utilizada por el traductor en su tarea mediadora. El encargo especifica (a veces nada más que implícitamente) las cualidades que el producto final del proceso traductor debe tener para cumplir los fines comunicativos ideados por el iniciador: la lengua (o, como en el caso de lenguas como el portugués, el castellano, el inglés y otras, la variedad lingüistica) en que debe estar escrito, el destinatario o los destinatarios a los que se dirigirá el texto, el medio por el que se transmitirá al destinatario (oral o escrito, y si escrito, por fax o por publicación en periódico, revista especializada, antología científica etcétera), el lugar y la fecha previstos para su publicación o recepción. Cuanto mayor la cantidad o especificidad de las informaciones proporcionadas, tanto más fácil será para el traductor producir una traducción que cumpla exactamente las finalidades deseadas. Si no sabe con suficiente exactitud para qué o para quién traduce, no le quedará más remedio que producir un texto "elástico" o "passe-par-tout" que sirve igualmente bien (o mal) para cualquier objetivo comunicativo pero que, lógicamente, no puede tener cualidades óptimas para un determinado fin específico.

No siempre es necesario que el iniciador especifique detalladamente el encargo de traducción. Hay casos en que la situación misma en que trabaja el traductor (por ejemplo, si es empleado de una empresa) o el propio texto base (por ejemplo, si se trata de unas instrucciones al uso de un aparato electrodoméstico que suelen traducirse para exactamente el mismo fin) no le dejan al traductor mucha opción porque existe lo que podríamos llamar un encargo rutinario o convencional. Un traductor experto dispondrá de los conocimientos práctico-profesionales que le permitirán tomar las decisiones pertinentes aun cuando el en- 
cargo no esté formulado con suficiente claridad. En cambio, en la formación de traductores, donde los alumnos todavía no son expertos en cuestiones profesionales, es conveniente que el encargo de traducción dado por el profesor sea lo más explícito posible, describiendo con suficientes detalles la situación comunicativa para la que se necesita el texto traducido - tanto más si se trata de un texto que no tiene encargo convencional, como es el caso de los artículos periodísticos utilizados con tanta frecuencia en las clases de traducción.

En todo caso, la primera fase del proceso de traducción suele ser la interpretacion del encargo de traduccion. 'Interpretar el encargo' significa sacar de él todas las informaciones que puedan guiar las decisiones del traductor en el proceso de traducción. El resultado de la interpretación será, por lo tanto, la definición de lo que, en la terminología de Hans J. Vermeer, se 1lama el "skopos" del proceso traductor (cf. Vermeer 1986: 6: "a finalidade da tradução").

Después de interpretar el encargo de traducción, el traductor procede al analisis del texto base, para el que se apoya en sus conocimientos del tema textual y la correspondiente terminología y en el dominio que tiene de la lengua y cultura del área socio-geográfica de la que procede el texto base. Si le faltan conocimientos o capacidades para lograr un análisis satisfactorio, tiene que recurrir a los métodos investigativos apropiados para procurárselos. En la didáctica de la traducción, es importante enseñarles a los alumnos los procedimientos analíticos (morfológicos, sintácticos, lingüístico-textuales y pragmáticos) necesarios para un análisis profundo de cualquier aspecto textual.

Comparando el resultado del análisis textual con el de la interpretación del encargo, el traductor puede esbozar una estrategia del transfer, es decir, puede optar por un determinado tipo de traducción (véase, por ejemplo, Nord 1997) que implica determinados procedimientos de reproducción, sustitución, adaptación, etcétera, dejándose guiar por sus conocimientos teórico-metodológicos acerca de la traducción o bien buscando las informaciones necesarios en la documentación traductológica existente. 
La cuarta fase del proceso consiste en la puesta en práctica de la estrategia elegida, produciendo un texto meta o TM (tradicionalmente, texto de llegada, término que también me suena demasiado a trayecto fijo que no permite más de un punto final) que corresponda a los requerimientos del encargo de traducción. En la produccion del texto meta, el traductor se vale de los conocimientos temático-terminológicos y linguo-culturales que tiene del área socio-geográfica al que va destinado el texto meta o, en caso de no tenerlos, los busca mediante los métodos investigativos adecuados.

Según esta concepción funcional del proceso de traducción, traducir es producir un texto en una lengua meta (TM) a base de un texto formulado en otra lengua (texto base, TB). El TM debe cumplir determinadas funciones comunicativas para los destinatarios pertenecientes a la cultura receptora, mientras que el texto original cumple (o cumplió) determinadas funciones comunicativas para unos destinatarios ubicados en la cultura base. Siguiendo las instrucciones del encargo de traducción, formuladas explícita o (en la mayoría de los casos) implícitamente, el traductor desprende las informaciones necesarias del texto base, procesándolas y reformulándolas hasta que tengan una forma aceptable para los receptores de la cultura meta. "Aceptable" será una forma que no impida o estorba la comprensión y que permita a los receptores utilizar el texto para los fines comunicativos que deseen. La aceptabilidad del producto se mide comparando el texto meta con las especificaciones del encargo de traducción.

En este proceso, el traductor necesita una serie de conocimientos (profesionales, temáticos, lingüísticos y culturales de las dos culturas entre las que va a mediar y también traductológicos en el sentido más amplio de la palabra). Si le faltan esos conocimientos, tiene que emplear los metodos investigativos adecuados para conseguirlos o completar los pocos que tenga. También es preciso que domine las técnicas de interpretación del encargo de traducción, de análisis y producción textuales y de planeamiento estratégico del transfer. Además necesita determinadas capacidades personales que le facilitarán su tarea traductora: capacidad de abstracción (para la comparación del texto original con las especificaciones del encargo de traducción), 
capacidad de decisión (para eligir una estrategia adecuada de entre varias que están a su disposición), capacidad de transfer (para poner en práctica la estrategia elegida) y capacidad crítica (para evaluar su propio producto frente a las exigencias del encargo de traducción. Estas capacidades - dicho sea de paso - podrian considerarse como condición previa para aprender a traducir; es muy dificil que una persona que no dispone de estas capacidades (al menos de una forma elemental) llegue a ser un buen traductor profesional. Es obvio que la tarea esencial de la formación profesional consistirá en el desarrollo progresivo de las capacidades personales y la enseñanza y aplicación de los conocimientos, procedimientos y técnicas respectivas.

Mientras éstas no se hayan desarrollado hasta un nivel suficiciente, el aprendiz de traductor puede tropezar con diversas dificultades debidas o bien a factores externos (p.ej., la imperfección lingüística o formal del TB) o bien a factores internos relacionados con las lagunas existentes en sus propios conocimientos, como por ejemplo:

- falta de conocimientos prácticos sobre la vida profesional de los traductores;

- insuficiencia de los conocimientos sobre la lengua y cultura base, por ejemplo en cuanto a

- vocabulario general, es decir, alguna palabra o giro que no se entiende, como en el caso de mis alumnos alemanes que no entendían la frase algo se está yendo de vareta en un texto español sobre el turismo de masas porque no conocían el giro idiomático irse de vara y tampoco lo encontraban en el diccionario porque no se habian dado cuenta de que vareta era un diminutivo de vara;

- estructuras gramático-sintácticas, es decir, alguna construcción morfológica o sintáctica que no se comprende porque, por ejemplo, en una oración larga los alumnos no se enteran de las relaciones de concordancia entre nombres y adjetivos;

- estilística y textualización, es decir, determinadas figuras retóricas desconocidas o el valor estilístico 
de ciertas características formales o lingüísticos interpretado como elemento original elegido por el autor para conseguir un efecto retórico porque el alumno no sabe que se trata de rasgos puramente convencionales del género al que pertenece el texto en cuestión; se incluyen aquí los modos específicos de composición y cohesión textual (recurrencia/sustitución pronominal/variación estilística, tema/ rema, perspectiva funcional de las oraciones, amáfora/catáfora, isotopía, etcétera) descubiertos por la lingüística del texto;

- comportamiento, es decir, formas de comportamiento verbal o no-verbal (gestos, ademanes, lenguaje del cuerpo, rituales etc.) que no se pueden interpretar o se malinterpretan porque se desconoce su significado o se confunden con formas de comportamiento análogas de la cultura meta (cf. Poyatos 1983);

- insuficiencia de los conocimientos temáticos y terminológicos, por ejemplo cuando

- el traductor no tiene suficientes conocimientos objetivos para comprender el mensaje del texto original llenando las lagunas presupositivas que el autor (experto) dejó porque estaba seguro de que su destinatario (experto) sabria llenarlas a base de sus conocimientos previos del tema;

- el traductor tiene el bagaje cultural necesario para comprender el mensaje general del texto, pero no los conocimientos específicos de terminología para entender las referencias técnicas o científicas;

- insuficiencia de los conocimientos traductológicos, cuando, por ejemplo,

- el traductor no dispone de los conocimientos teórico-metodológicos necesarios para esbozar una estrategia traductora coherente;

- el traductor no se entera de los problemas de traducción o, si se entera, no sabe resolverlos; 
- el traductor desconoce las fuentes de documentación y/o los procedimientos investigativos para compensar sus deficiencias cognitivas o metodológicas;

- insuficiencia de los conocimientos de la lengua y cultura meta, por ejemplo en cuanto a

- vocabulario general (morfología, colocaciones, fraseología etc.),

- estructuras gramaticales o sintácticas (subjuntivo, tiempos del verbo, conjugación/declinación),

- estilística y textualización general o de determinados tipos de texto,

- convenciones de comportamiento verbal y no verbal.

Existen varias fuentes de documentación e investigación para compensar algunos de estas deficiencias, y su uso adecuado debe enseñarse en la formación de traductores. Pero no todas ofrecen la información necesaria de una manera satisfactoria o en cantidad suficiente, y para varios problemas no hay solución fácil.

- Los conocimientos prácticos de la profesión, que ayudan al traductor no sólo en la fase de interpretación del encargo de traducción sino también en la de evaluación del producto, sólo pueden adquirirse en una formación de traductor profesional, sea "on the job" y preguntando a los colegas experimentados o bien en las facultades o instituciones de enseñanza de la traducción profesional, si esta enseñanza está modelada conforme a la práctica profesional, con respecto a la cultura base así como a la cultura meta: con encargos realistas, textos que se traducen profesionalmente, períodos de trabajo práctico en empresas o agencias de traducción etcétera. Los manuales de traducción deberían incluir también este aspecto tan esencial para un buen traductor porque los alumnos tienen que aprender cómo formular las preguntas adecuadas para conseguir la información deseada de los expertos profesionales y también de los clientes y encarga- 
dores de traducción. La literatura traducto-lógica hasta ahora no es muy abundante sobre este tema tan ligado a la práctica, al menos es difícil encontrar la información rápidamente en un momento concreto, porque se encuentra dispersa en articulitos en revistas o antologías. Lo que falta todavía es un compendio sistemático que contenga la información al respecto.

- Para completar los conocimientos lingüístico-culturales en ambas culturas se emplean sobre todo diccionarios (normalmente monolingües para el análisis del texto base, bilingües y monolingües para el uso activo de la lengua meta) y manuales de gramática y sintaxis de las lenguas base y meta. Sin embargo, los traductores muchas veces se quejan de la insuficiencia de los diccionarios bilingües, en los que no se indican los contextos donde las palabras se usan con mayor frecuencia, las colocaciones y fraseologismos de los que forman parte, las restricciones de uso, sean estilísticas, regiolectales, sociolectales etc., y también de las gramáticas, que suelen describir el sistema lingüístico y no el uso de la lengua. Para la traducción alemán-español, por ejemplo, sería importante conocer los tipos de texto y los contextos en los que se usa el gerundio con más frecuencia que una oración subordinada.

Además, el traductor precisa de una buena capacidad de producir textos en la lengua meta (que no es siempre su lengua nativa) y tiene que dominar los diversos aspectos de esta capacidad redactora. En este caso, las lagunas y insuficiencias de competencia no pueden compensarse tan fácilmente como en la fase de recepción. Algunos manuales de estilo y de redacción (para usuarios de lengua materna) empiezan a fijarse en estos aspectos del uso lingüístico (véase, por ejemplo, Fernández de la Torriente 1991, que abarca los géneros de descripción, narración, ensayo, reportaje, carta, informe, reseña y exposición científica etc.).

Sin embargo, hay que destacar que las deficiencias cognitivas en cuanto a vocabulario, gramática o textualización 
nunca deben ser tales que impidan la comprensión o la composición general del texto; la enseñanza de traducción no debe empezar hasta que alumnos hayan alcanzado un nivel de conocimientos lingüístico-culturales que les permita entender los textos base sin mayores dificultades. La clase de traducción no es el lugar adecuado para el desarrollo básico de la competencia lingüística en la lengua extranjera, aunque ésta se vaya perfeccionando con cada texto que se traduce.

- Los conocimientos temáticos y terminológicos necesarios para entender el texto base y formular un texto meta adecuado tampoco deberían formar parte de la enseñanza de traducción sino que deberian transmitirse en clases específicas. Esto no vale sólo para la traducción técnica o científica, cuya enseñanza deberia ir coordenada con las clases de economía, derecho, ingeniería, informática etc., sino también para la traducción de los llamados textos generales. Es un hecho ampliamente comprobado que las deficiencias lingüistico-culturales dejan de constituir un obstáculo a la comprensión textual si el lector ya dispone de antemano de los conocimientos temáticos y del bagaje cultural y general correspondientes que le facilitan el procesamiento de las informaciones ofrecidas por el texto. Por lo tanto, sería útil que también los textos generales empleados como textos base fueran acompañadas de fuentes de información enciclopédica sobre el tema en cuestión, al menos en las primeras fases de la enseñanza de traducción.

También en lo que se refiere a la terminología, la información ofrecida por las fuentes de información, por ejemplo los diccionarios especializados, los glosarios y los bancos de datos terminológicos no siempre es fiable, sobre todo si se trata de fuentes bilingües. Es bien sabido que para evaluar la equivalencia conceptual de dos términos, a veces es necesario analizar a fondo la literatura especializada con las definiciones y los contextos correspondientes. Además, los procedimientos terminográficos no son universales sino también pueden ofrecer rasgos 
específicos en las dos culturas implicadas. Si la literatura temática consultada se da tanto en la lengua original como en el idioma meta, el traductor puede matar dos pájaros de un tiro: no solamente saca de ella informaciones objetivas relacionadas con el tema del texto sino que muchas veces los textos enciclopédicos le ofrecen también informaciones terminológicas que le serán útiles en la fase productiva del texto meta.

El esquema siguiente (ESQUEMA 2) presenta una sinopsis de los conocimientos necesarios en cada una de las fases del proceso de traducción así como de las fuentes orales o escritas existentes para compensar las insuficiencias al respecto. La última columna se refiere a las habilidades investigativas que en cada caso han de desarrollarse en la formación profesional. Hemos introducido unos signos de interrogación para indicar las áreas que todavía quedan por elaborarse. 
2. Conocimientos y habilidades necesarias en el proceso de traducción

\begin{tabular}{|llll|}
\hline $\begin{array}{l}\text { Fase de } \\
\text { proceso de } \\
\text { traducción }\end{array}$ & $\begin{array}{l}\text { Conocimientos } \\
\text { necesarios }\end{array}$ & $\begin{array}{l}\text { Fuentes escritas } u \\
\text { orales o modo de } \\
\text { transmisión de } \\
\text { conocimientos }\end{array}$ & $\begin{array}{l}\text { Habilidades } \\
\text { investigativas } \\
\text { necesarias }\end{array}$ \\
\hline \begin{tabular}{llll}
\hline INTERPRETACIÓN \\
DEL ENCARGO DE \\
TRADUCCIÓN
\end{tabular} & $\begin{array}{l}\text { práctica de la traduc- } \\
\text { ción profesional en la } \\
\text { cultura del iniciador }\end{array}$ & $\begin{array}{l}\text { enseñanza "realista“, } \\
\text { tutoría por traductores } \\
\text { profesionales }\end{array}$ & $\begin{array}{l}\text { formular preguntas } \\
\text { adecuadas }\end{array}$ \\
\hline
\end{tabular}

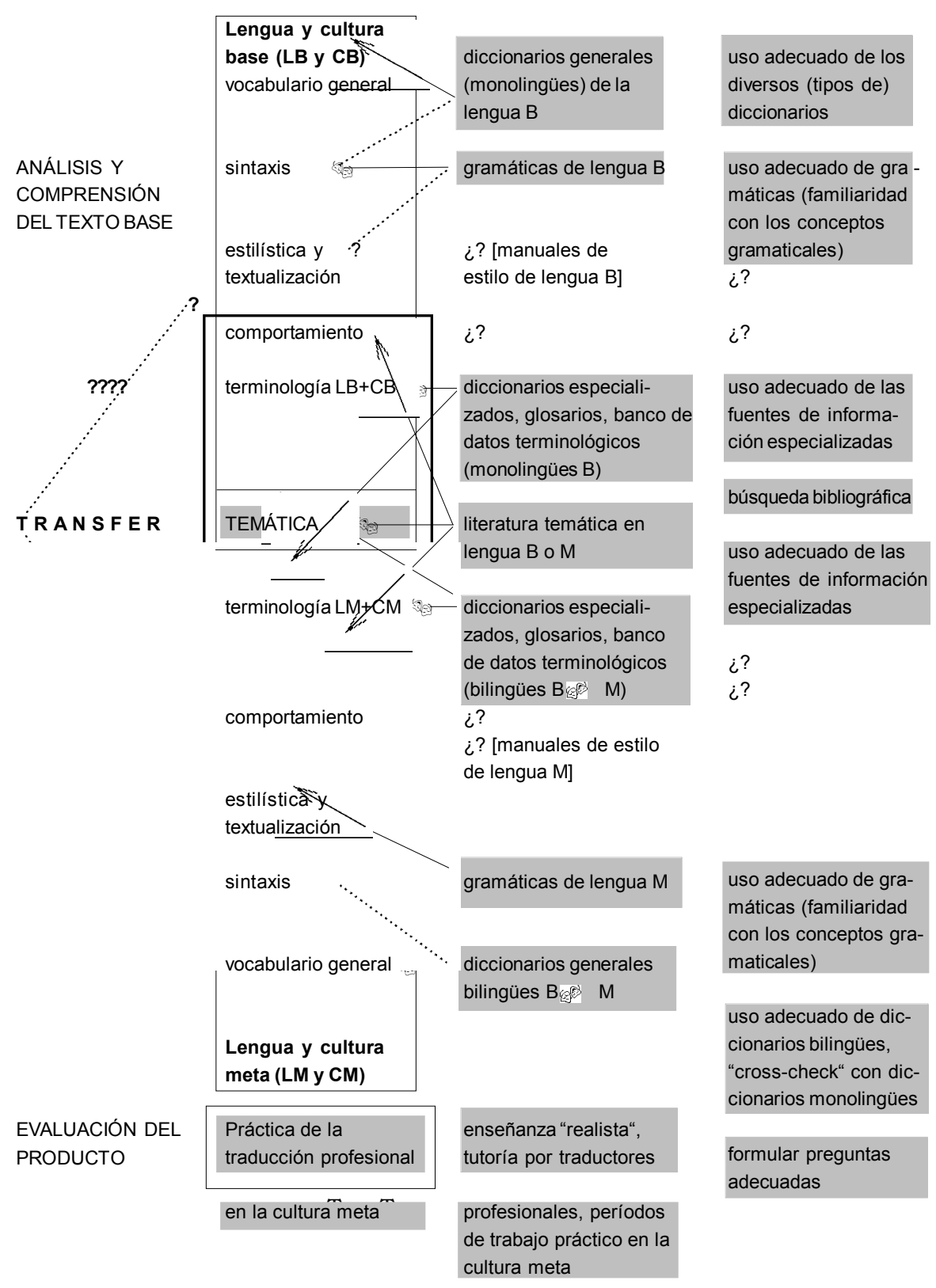


El esquema 2 muestra que existen dos áreas en las que las fuentes de información son escasas o nulas. Uno es el campo del comportamiento tanto verbal (incluyendo la estilística y textualización) como no verbal, cuya investigación corresponde, en primer lugar, a la filología (en el sentido amplia de la palabra) o más bien culturología unilateral. Lo que hace falta - tanto para la práctica profesional como para la enseñanza de traducción es una descripción lo más exhaustiva posible de los comportamientos específicos de las culturas enfrentadas en la traducción. Permítanme que lo explique mediante una pequeña anécdota personal:

Cuando hace muchos años empecé a estudiar el castellano, aprendi que danke "significa" gracias. Y a partir de entonces utilicé gracias en España o Latinoamérica en las situaciones análogas a las que, en Alemania, hubieran exigido decir danke, contribuyendo asi, sin quererlo, al estereotipo generalizado de que "los alemanes" suelen dar las gracias (o también pedir perdón) a cada paso. Porque nadie me enseñó que hay bastantes situaciones en que un alemán daría las gracias y un espanol, no - sin que se le reprochara una falta de educación.

El ejemplo ya destaca el segundo desiderátum, que es auténtica-mente traductológico: No sólo carecemos de estudios unilaterales sobre el comportamiento específico preferido en una determinada cultura, sino también de investigaciones comparativas que contrasten las formas de comportamiento de una cultura con las de otra, fijándose no sólo en la diferencia de formas (danke o gracias) sino también en las diferencias de frecuencia (cuántas veces se dice danke o gracias) y distribución (cuáles son las situaciones en las que se dice danke o gracias). Porque son la frecuencia del uso y la distribución contextual las que, al fin y al cabo, determinan el significado que un determinado comportamiento tiene para los miembros de una cultura en una situación dada.

Consideraciones análogas pueden aplicarse a las áreas lingüísticas mencionadas arriba. Al traductor no le sirven las gramáticas contrastivas que comparan los sistemas lingüisticos 
de dos lenguas (confrontando, por ejemplo, el uso del subjuntivo en castellano y alemán, véase, por ejemplo, Cartagena/Gauger 1989); lo que necesita es una comparación de las hablas de dos comunidades culturales (y hay que tener en cuenta que las culturas no siempre se corresponden con las áreas lingüísticas ni tampoco con las comunidades políticas), una confrontación de los usos estilísticos y de textualizacón y de los tipos de texto. Semejantes estudios comparativos deberían basarse en amplios corpus de textos de los más diversos géneros, analizados, en primer lugar, unilateralmente (en lo que Harald Weinrich ha llamado "estilística textual", cf. Weinrich 1982, 1993) y contrastados, después, en una confrontación bilateral o, mejor, multilateral. La base de la comparación, o tertium comparationis, debería ser la función-en-situación (véase el análisis contrastivo de títulos alemanes, ingleses, franceses y españoles en Nord 1993, resumido brevemente en castellano en Nord 1994 y en inglés en Nord 1995).

Este Manual Comparativo de de los Tipos Textualesqueda por escribirse, y como tendrá que basarse en numerosos corpus compuestos de grandes cantidades de textos para contar con una sólida base estadística, será cosa de años, por no decir decenios. Mientras tanto, los traductores y los alumnos de las instituciones formadoras tienen que contentarse con unos instrumentos menos exigentes y más accesibles: los textos auxiliares.

Grosso modo, los textos auxiliares son textos de los que se pueden sacar las informaciones necesarias para producir buenas traducciones que sirvan para cumplir las funciones comunicativas deseadas. La idea de aprovecharse de textos ya existentes utilizándolos como modelos para el texto meta pedido por el iniciador, se basa en el hecho de que, en materias de comunicación, "no hay nada nuevo bajo el sol". Los textos que tenemos que producir ya existen, o al menos existen piezas "prefabricadas", módulos que podemos utilizar en la producción de un texto. No hay textos radicalmente "nuevos", son siempre las mismas situaciones en las que nos comunicamos, y para facilitarnos la comunicación solemos utilizar textos o segmentos de textos formados según modelos preexistentes. 
Al comparar los textos de dos culturas diferentes, contamos con las siguientes variables:

- Tema: un texto sobre las consecuencias perjudiciales del turismo de masas puede tener características diferentes de un texto sobre la cría de caballos (al menos no sabemos si el tema es una variable relevante hasta que no lo hayamos comprobado mediante un análisis de corpus).

- Género o tipo de texto: un ensayo filosófico es distinto de unas instrucciones para el uso de un aparato electrodoméstico.

- Destinatario: una receta para el ama de casa se distingue de una receta para niños.

- Afiliaciones históricas, regionales, sociales, etc.: un comentario periodístico escrito para un diario argentino se diferencia de uno escrito para un periódico español, un texto escrito por un ingeniero para ingenieros se distingue de un texto escrito por un cocinero para otros cocineros.

Partimos de la hipótesis de que existe una correlación entre las variables mencionadas y los rasgos intratextuales o lingüísticos específicos (composición, medios de cohesión, léxico, sintaxis, etc.) de un texto. Al comparar dos textos escritos en dos lenguas diferentes, las diferencias intratextuales sólo pueden atribuirse al factor lengua si todas las variables se mantienen constantes. Poniéndolo al revés: Comparando una receta española para amas de casa y una receta inglesa para niños, no sabemos cuáles diferencias intratextuales entre los dos textos se deben al contraste pragmático entre los dos destinatarios y cuáles están determinadas por el contraste lingüístico castellano-inglés.

Esto significa: Cuanto mayor el número de factores extratextuales constantes, tanto más fácil será la correlación entre una variable extratextual y las características intratextuales específicas del texto en cuestión.

Según las variables mantenidas constantes en la comparación, podemos distinguir varios tipos de texto auxiliar en la 
investigación traductológica: A primera vista, parece que el mayor número de constantes se da en la comparación de un texto base con "su" traducción porque no existirán otros dos textos que tengan en común hasta los más pequeños detalles de contenido (véase Wotjak 1987: 67). Por eso, algunos autores mantienen que el original y la traducción formarian la pareja ideal para la comparación interlingual de textos y que son, por lo tanto, textos paralelos. Por otro lado, las situaciones de un texto auténtico y su traducción a otra lengua y cultura no son tan parecidas como parece. Por regla general (salvo en el caso de la interpretación simultánea) difieren en cuanto a tiempo y lugar (los textos traducidos suelen llegar a sus destinatarios más tarde que los originales y en una situación de la cultura meta), muchas veces también en cuanto a intención comunicativa (la traducción puede servir, por ejemplo, para informar sobre el texto base y entonces tendrá una intención metatextual aunque el texto base haya tenido una intención objetual). Pero se debe a la propia definición de traducción que los dos textos siempre se distinguen en cuanto a las condiciones de su origen (una traducción es un texto formulado a base de otro, y el traductor no es un autor sui generis) y también en cuanto al destinatario. Aunque éste sea la más fiel réplica del destinatario del TB en lo que se refiere a edad, sexo, educación, intereses etcétera, el destinatario de una traducción se diferenciará del de un original por haber crecido y formarse en un ambiente sociocultural diferente. Tiene otro bagaje cultural, otro "horizonte", otra perspectiva hacia el mundo, otros sistemas de valores, otras tradiciones, por lo que sus presuposiciones al recibir un determinado texto son, en esencia, también otras. Está claro que en un caso concreto, sobre todo cuando se trata de dos culturas vecinas o históricamente ligadas, puede ocurrir que esta diferencia esencial no se manifiesta muy claramente, pero aún así, comparando un texto y su traducción no podemos estar nunca seguros de si los rasgos que los separan son consecuencia de estas diferencias situacionales o si de verdad se deben al contraste lingüístico-cultural.

Aunque no niego el valor de una comparación entre un texto base y su(s) traduccion(es) en la enseñanza de traducción (demostrada, por ejemplo, por Wilss 1996a y 1996b), creo que un 
texto traducido no puede servir de modelo para la producción del texto meta. Siguiendo a Snell-Hornby (1988: 86) limitaremos, por lo tanto, el concepto de texto paralelo a "two linguistically independent products arising from an identical (or very similar) situation", es decir, dos (o más) textos auténticos originados en culturas distintas, pero en situaciones comunicativas que muestran el mayor número posible de variables constantes.

Fueron Sager y McDonald los que ya en 1973 comprobaron que el análisis comparativo de textos paralelos constituye un instrumento valioso en la enseñanza de lenguas extranjeras (véase Sager/McDonald 1973: 181). En la traductología didáctica, son sobre todo Holz-Mänttäri (1981), Snell-Hornby (1988), Kußmaul (1978), Thiel (1985) y, más recientemente, Toury (1995) y Wilss (1996a, 1996b) los que destacan la importancia de los textos paralelos para el trabajo del traductor (aunque Wilss incluye aquí la comparación entre texto base y texto meta). Toury describe la función de los textos paralelos de la siguiente manera:

Due to their habitual use, they [the two texts, C.N.] may be regarded as codified entities: while never ceasing to be texts, they also constitute elements of certain repertoires pertaining to the two cultures in question. And since the two are used in comparable situations, they can be taken as functionally parallel, hence initially interchangeable with respect to the situation at hand (Toury 1995: 89s.).

En nuestro caso, el texto paralelo es, como lo formula Thiel (1985: 121), "the sought-after text", el texto buscado, en la lengua y cultura meta, es decir un modelo textual que corresponde a las exigencias del encargo de traducción. Por regla general, un texto paralelo es un texto auténtico de la cultura meta que pertenece al mismo género textual, lo que significa que se usa para los mismos fines comunicativos como el texto base. Cuanto más se parecen los temas, tanto mejor. Si un texto paralelo no sólo corresponde al mismo género y tema sino que también tiene el mismo contenido, podemos incluso hablar de un texto modelo, como en el ejemplo siguiente. Se trata de las instrucciones de preparación de un pudin de vainilla. El producto español no es 
un flan auténtico sino que contiene exactamente los mismo ingredientes como el producto alemán.

EJEMPLO 2

Flan Potax

\section{INSTRUCCIONES}

De MEDIO LITRO de leche fría se separa una taza en la que se disuelve el contenido de este sobre.

Se pone a hervir el resto de la

POLAK-Pudding Vanille-Geschmack

\section{Für die Zubereitung als Pudding} benötigen Sie:

$1 / 21$ Milch und 2 Eßlöffel Zucker (40g)

1. Von der Milch 6 Eßlöffel abnehmen und damit das Puddingpulver gut anrühren.

leche con azúcar (de 4 a 6 cucha- 2. Die übrigen Milch mit dem Zucker radas). zum Kochen bringen.

Al comenzar a hervir se vierte el contenido de la taza y se remueve constantemente.

Tan pronto como espese se retira delfuego, se vierte en un molde bastante caramelizado y se deja enfriar.
3. Die Milch von der Kochstelle nehmen, das angerührte Puddingpulver hineingeben und unter ständigem Rühren 1 Minute aufkochen lassen. 4. Den fertigen Pudding in eine mit kaltem Wasser ausgespülte Form füllen und kaltstellen. Später stürzen.

Los textos son textos modelo uno para el otro. Además de las diferencias lingüísticas (por ejemplo, formas impersonales reflexivas en el texto español - infinitivos en alemán) se notan algunas diferencias formales (p.ej., la indicación separada de los ingredientes adicionales y la numeración en el texto alemán) y culturales (p.ej. la forma de medir la cantidad de leche separada: una taza - 6 cucharadas, la cantidad de azúcar añadida: 4 a 6 cucharadas - 2 cucharadas o 40 gramos, y la caramelización del molde para el pudin español, que es lo único que recuerda al flan). Si tiene que traducir el texto español al alemán (o viceversa), el traductor puede imitar las formas lingüísticas y estructurales del otro texto. En cuanto a las diferencias culturales, depende del encargo de traducción si tiene que decidirse o bien por una estrategia imitativa o bien por una estrategia adaptiva. $\mathrm{Si}$ opta por la estrategia imitativa, debe tener en cuenta las expectativas de los respectivos destinatarios, a los que quizás no les 
guste un pudin con tanto (o con tan poco) azúcar o quienes esperan otro producto si se les anuncia un "flan", como demuestran los siguientes ejemplos. El texto alemán, que describe la preparación de un flan de verdad, no es precisamente un texto paralelo al "Potax", porque se diferencia en cuanto al medio transmisor. Pero como tiene algunas semejanzas en cuanto a situación, intención, tema y función textual (género: receta), podríamos considerarlo como texto vecino. El texto español "Flan de huevos", a su vez, es un texto paralelo al "Creme Caramel".

EJEMPLO 3

Creme Caramel

1/4 $l$ Milch, 150ccm Sahne, 3 Eier, 2 Eßsl. Zucker; für den Karamel: $100 \mathrm{~g}$ Zucker.

Milch und Sahne in einem Topf lauwarm werden lassen. Inzwischen Eier und Zucker so lange schlagen, bis sich der Zucker aufgelöst hat. Die warme Milch hineinrühren und zur Seite stellen. Den Zucker für den Karamel mit $50 \mathrm{ccm}$ Wasser bei schwacher Hitze langsam schmelzen lassne. Dann die Temperatur erhöhen und, ohne zu rühren, so lange kochen, bis der Sirup goldbraun ist. Den Karamel schnell in vier kleine mit kaltem Wasser ausgespülte Puddingförmchen gießen und mit der Eiermilch auffüllen. Die Förmchen in die zur Hälfte mit heißem Wasser gefüllte Fettpfanne des Backofens stellen. Bei $180 \mathrm{Grad} / \mathrm{Gas}$ Stufe 3 etwa 40 Minuten backen, bis die Creme fest geworden ist. Die fertige Creme kühl stellen. Vorsichtig auf kleine Teller stürzen. (Ohne Kühlzeit 1 Stunde)

Dieses Rezept ist für 4 Personen berechnet.

(Sacado de la revista femenina alemana Brigitte, 1986)

EJEMPLO 4

FLAN DE HUEVOS

Cantidades: Para 4 personas

Tiempo de cocción: Media hora

Ingredientes: Medio litro de leche; 4 huevos; 1 bastón de vainilla; 100 gramos (1/4 lb.) de azúcar.

Se baten bien los cuatro huevos y se les añade el medio litro de leche tibia y la vainilla, así como el azúcar, del que se aparta una cucharada.

Se toma un molde (flanera) y se carameliza el fondo poniéndolo al fuego con la cucharada de azúcar apartada. Cuando está moreno, se vierte en el molde la mezcla preparada, retirando la vainilla.

Se cuece el flan en el horno bien caliente. Cuando está cocido (se comprueba su cocción pinchándolo con una aguja de hacer media, que ha de salir limpia si está cocido), se saca del horno y se deja enfriar.

TradTerm, 4(1), $1^{\circ}$ semestre de 1997 , p. 101-124 
Se vuelca entonces el flan sobre un plato o fuente y se sirve frío.

Puede también cocerse al baño maría, es decir, poniéndose el molde en una cacerola más grande que él, que contenga agua que vaya hirivendo, procurando que no entre en la flanera. El flan irá cuajando y cociéndose igualmente.

(G. Bernard: Un menú para cada dia. Barcelona 1962)

Comparando los dos textos, se ve que otra vez hay muchos rasgos lingüísticos similares y otros diferentes. Es evidente que los textos paralelos ofrecen también mucho material utilísimo en cuanto a la terminología adecuada, que precisamente en el caso del arte culinario es muy dificil de encontrar en los diccionarios bilingües generales. Sin embargo, una golondrina no hace verano, como se dice, y un solo texto paralelo o incluso modelo no constituye todavia una base material suficientemente fiable para servir de norma traductora. No sabemos si el texto paralelo elegido es de verdad un representante típico o incluso prototípico del género en cuestión, así que el traductor debe ir con sumo cuidado al utilizar estos textos auxiliares. En todo caso sería conveniente basarse en un corpus de varios textos paralelos para ver cuál de ellos puede servir de modelo para la traducción.

El texto "Creme Caramel" va acompañado de una nota a pie de página que explica el origen y las características específicas del flan:

Die Creme Caramel, in Spanien "flan" genannt, gehört zu den klassischen Nachspeisen der südeuropäischen Küche. Der Karamel - das ist geschmolzener gebräunter Zucker - bildet eine Glasur auf einer Creme aus Eiern, Milch und Zucker.

(La Creme Caramel, llamado "flan" en España, es uno de los postres clásicos de la Europa del Sur. El caramelo - azúcar hecho almíbar a fuego lengo hasta que se ponga moreno - forma una capa glaseada sobre una crema cocida de huevos, leche y aczúcar. Trad. C.N.)

Se ve que este texto pertenece a otro género que las recetas anteriores. Ofrece unas informaciones enciclopédicas sobre el objeto referencial del que tratan los textos instructivos. No es, por lo tanto, un texto paralelo, pero es también un texto auxiliar 
porque puede servir, por ejemplo, a una persona que quiere traducir la receta "Creme Caramel" al castellano (por ejemplo, en lo que se refiere a la traducción del nombre del producto). Por eso, este texto podría considerarse como un texto de información enciclopédica o texto informativo (en inglés: background text, en alemán: Hintergrundtext).

Para concluir, volvamos a mirar el esquema 2. Los signos de interrogación marcan aquellos aspectos del proceso traductor para los que no existen todavia fuentes de información o documentación adecuadas para ayudar al traductor en su tarea de producir un texto meta funcional que pueda competir con los textos existentes de la cultura meta (si es esto lo que exige el encargo de traducción). Un desiderátum para el futuro de la traductología aplicada sigue siendo una comparación empírica y basada en unos corpus amplios de la mayor cantidad de tipos textuales posible. Hasta que no se cumpla este deseo común de traductores, traductólogos, enseñadores y aprendices de traducción, tenemos que contentarnos con lo que existe, y no es poco: todo el acervo de textos existentes de la cultura meta nos puede servir, de una u otra forma, como material auxiliar en la tarea de traducir, sea como modelo más o menos exacto de la meta que se quiere o debe conseguir, sea como punto de referencia para medir la distancia entre las dos culturas, si el encargo de traducción exige que la traducción refleje precisamente lo que es "diferente" en el texto (o la cultura) base, sea como fuente de información sobre el objeto textual.

Hemos visto que hay varios tipos de textos auxiliares que nos pueden ofrecder ayuda en diferentes problems de traducción. Los textos paralelos pueden guiar al traductor en lo que se refiere a convenciones estilísticas generales, textuales o de comportamiento (por ejemplo, convenciones de pesos y medidas), así como en la terminología (por ejemplo, stürzen - volcar, de un flan, traducción que el diccionario bilingüe alemán-español ofrece entre derribar, echar abajo, tumbar, arrojar, precipitar, hacer caer etcétera, sin referencia alguna a la terminología culinaria) o fraseología (por ejemplo, zum Kochen bringen-poner a hervir). Los textos modelo pueden imitarse incluso en cuanto a su macro y microestructura o sus presuposiciones culturales (por ejemplo, 
si hay que explicarles a los destinatarios la técnica de caramelizar un molde o no). Los textos vecinos ponen de manifiesto las características textuales no ligadas al tema o al contenido específico sino más bien a la función textual (por ejemplo, receta de cocina o texto instructivo en general). $\mathrm{Y}$ los textos informativos o enciclopédicos ofrecen informaciones temáticas que le facilitan al traductor no experto en el campo la comprensión incluso de textos muy especializados escritos por expertos para expertos.

En las primeras fases de la enseñanza de traducción, parece conveniente que el profesor traiga los textos auxiliares explicando la mejor manera de aprovecharse de ellos y también las limitaciones de su utilización. En una fase avanzada, los alumnos buscarán ellos mismos los textos auxiliares adecuados, lo que ya los prepara mejor para la vida profesional de traductor.

\section{Referências Bibliográficas}

CARTAGENA, Nelson \& Hans-Martin Gauger (1989). Vergleichende Grammatik Spanisch-Deutsch, 2 vols. Mannheim etc., Duden.

FERNÁNDEZ DE LA TORRIENTE, Gastón (1991). Cómo escribir correctamente. La comunicación escrita. 17a ed. Madrid, Playor.

HOLZ-MÄNTTÄRI, Justa (1981). Hilfsmittel des Übersetzens. Mitteilungsblatt für Dolmetscher und Übersetzer (MDÜ) 5, 27, 8-9.

NORD, Christiane (1991). Text Analysis in Translation. Amsterdam/ Atlanta: Rodopi. Trad. inglesa de Textanalyse und Übersetzen, Heidelberg: Groos ${ }^{1} 1988,{ }^{3} 1995$.

NORD, Christiane (1993). Einführung in das funktionale Übersetzen. Am Beispiel von Titeln und Überschriften. Tübingen-Basel, Francke.

NORD, Christiane (1994). Las funciones comunicativas y su realización textual en la traducción. In: Sendebar 5, 1994, 85-103.

NORD, Christiane (1995). Text-Functions in Translation. Titles and Headings as a Case in Point. In Target 7, 2, 261-284.

NORD, Christiane (1996a). El enfoque funcionalista de la traducción. In Voces 22, 12-20.

NORD, Christiane (1996b): Wer nimmt denn mal den ersten Satz? Überlegungen zu neuen Arbeitsformen im Übersetzungsunterricht.

TradTerm, 4(1), $1^{\circ}$ semestre de 1997 , p. 101-124 
In: LAUER, Angelika et al.: Übersetzungswissenschaft im Umbruch. Festschrift für W. Wilss zum 70. Geburtstag, Tübingen, Narr, 313-327. NORD, Christiane (1997). Translating as a Purposeful Activity. Functionalist Approaches Explained. Manchester, St. Jerome.

POYATOS, Fernando (1983). New Perspectives in Nonverbal Communication. Studies in Cultural Anthropology, Social Psychology, Linguistics, Literature, and Semiotics. Oxford.

SAGER, J. \& P.F. MCDONALD (1973). The Stylistic Comparative Analysis of Journalistic Texts as an Instrument of Cognitive Language Teaching. In: Zeitschrift für Amerikanische Linguistik 21, 180-200.

THIEL, Gisela (1985). Parallel Text Production. An Alternative in Pragmatically-Oriented Foreign Languiage Courses. In C. TITFORD $\&$ A.E. HIEKE (eds.). Translation in Foreign Language Teaching and Testing. Tübingen, Narr, 117-133.

TOURY, Gideon (1995). Descriptive Translation Studies and Beyond. Amsterdam-Philadelphia, Benjamins.

VERMEER, Hans (1986). Esboço de uma teoria da traduçâo. Porto, Edições Asa.

WEINRICH, Harald (1982). Textgrammatik der französischen Sprache. Stuttgart, Klett.

WEINRICH, Harald (1993). Textgrammatik der deutschen Sprache. Mannheim, Duden.

WILSS, Wolfram (1996a). Knowldege and Skills in Translator Behavior. Amsterdam-Philadelphia, Benjamins.

WILSS, Wolfram (1996b). Übersetzungsunterricht. Eine Einführung. Tübingen, Narr.

WOTJAK, Gerd (1987). Einzelsprachliches und Übereinzelsprachliches in der Bedeutung lexikalischer Einheiten (LE). In Linguistische Arbeitsberichte 61, 2-23. 